\title{
Curvature Inequalities between a Hessian Manifold with Constant Curvature and its Submanifolds
}

\author{
Münevver Yıldırım Yılmaz* and Mehmet Bektaş
}

(Communicated by Kazım İLARSLAN)

\begin{abstract}
In this paper after a short description of Hessian manifolds, we establish new curvature inequalities between a Hessian manifold and its submanifolds.

Keywords: Hessian Manifold;Riemannian Curvature Tensor; sectional curvature; spaceform.

AMS Subject Classification (2010): Primary: 53A15 ; 53C25: ; 53C05;53B05.

${ }^{*}$ Corresponding author
\end{abstract}

\section{Introduction}

Among the many Riemannian metrics that may exist on a flat manifold, Hessian metrics are the most compatible with the flat structure. A Riemannian metric on a flat and affine manifold is called a Hessian metric if it is locally expressed by the Hessian of functions with respect to the affine coordinate systems. A pair of a flat structure and a Hessian metric is called Hessian structure and a manifold equipped with a Hessian structure is said to be a Hessian manifold. Typical examples of of these manifolds are regular convex cones and the space of all positive definite real symmetric matrices [1-3] Hirohiko Shima introduced Hessian sectional curvature and its relations with Kaehlerian manifold. He also proved the theorems and gave important remarks on the spaceform of Hessian manifolds. In the light of these studies Yi ldiri $\mathrm{m}$ Yilmaz and Bektaş obtained some curvature conditions, results and integral inequalities on this type of manifolds, $[4,5,8]$

In this paper we focus on some new curvature estimates on Hessian manifolds and its submanifolds analogous with $[6,7]$.

\section{Preliminaries}

Let $M^{m}$ be a Hessian manifold with Hessian structure $(D, g)$. We express various geometric concepts for the Hessian structure $(\mathrm{D}, \mathrm{g})$ in terms of affine coordinate system $x^{1}, \ldots, x^{m}$ with respect to $\mathrm{D}$, i.e $D d x^{i}=0$

i) The Hessian metric ;

$$
g_{i j}=\frac{\partial^{2} u}{\partial x^{i} \partial x^{j}}
$$

ii ) Let $\gamma$ be a tensor field of type $(1,2)$ defined by

$$
\gamma(X, Y)=\nabla_{X} Y-D_{X} Y
$$

where $\nabla$ is the Riemannian connection for $g$. Then we have

$$
\gamma_{j k}^{i}=\Gamma_{j k}^{i}=\frac{1}{2} g^{i r} \frac{\partial g_{r j}}{\partial x^{k}}
$$

Received : 14-July-2016, Accepted : 27-February-2017 


$$
\begin{gathered}
\gamma_{i j k}=\frac{1}{2} \frac{\partial g_{i j}}{\partial x^{k}}=\frac{1}{2} \frac{\partial^{3} u}{\partial x^{i} \partial x^{j} \partial x^{k}} \\
\gamma_{i j k}=\gamma_{j i k}=\gamma_{k j i}
\end{gathered}
$$

where $\Gamma_{j k}^{i}$ are the Christoffel 's symbols of $\nabla$.

iii )Let $Q$ be a tensor field of type $(1,3)$ defined by

$$
Q=D_{\gamma}
$$

and call it the Hessian curvature tensor for $(D, g)$. Then we have

$$
\begin{gathered}
Q_{j k l}^{i}=\frac{\partial \gamma_{j l}^{i}}{\partial x^{k}} \\
Q_{i j k l}=\frac{1}{2} \frac{\partial^{4} u}{\partial x^{i} \partial x^{j} \partial x^{k} \partial x^{l}}-\frac{1}{2} g^{r s} \frac{\partial^{3} u}{\partial x^{i} \partial x^{k} \partial x^{r}}-\frac{\partial^{3} u}{\partial x^{j} \partial x^{l} \partial x^{s}} \\
Q_{i j k l}=Q_{i l k j}=Q_{k j i l}=Q_{j i l k}=Q_{k l i j} .
\end{gathered}
$$

iv ) The Riemannian curvature tensor for $\nabla$ is given by ;

$$
\begin{gathered}
R_{j k l}^{i}=\gamma_{r k}^{i} \gamma_{j l}^{r}-\gamma_{r l}^{i} \gamma_{j k}^{r}, \\
R_{i j k l}=\frac{1}{2}\left(Q_{j i k l}-Q_{i j k l}\right) .
\end{gathered}
$$

Definition 2.1. For a non-zero contravariant symmetric tensor $\xi_{x}$ of degree 2 at $x$, we set

$$
h\left(\xi_{x}\right)=\frac{\left\langle\varsigma\left(\xi_{x}\right), \xi_{x}\right\rangle}{\left\langle\xi_{x}, \xi_{x}\right\rangle}
$$

and call it the Hessian sectional curvature in the direction $\xi_{x}$, [1].

Theorem 2.1. Let $(M, D, g)$ be a Hessian manifold of dimension $\geq 2$. If the Hessian sectional curvature $h\left(\xi_{x}\right)$ depends only $x$ then $(M, D, g)$ is of constant Hessian sectional curvature. $(M, D, g)$ is of constant Hessian sectional curvature $c$ if and only if

$$
Q_{i j k l}=\frac{c}{2}\left(g_{i j} g_{k l}+g_{i l} g_{k j}\right)
$$

Corollary 2.1. If a Hessian manifold $(M, D, g)$ is a space of constant Hessian sectional curvature $c$, then the Riemannian manifold $(M, g)$ is a space of constant sectional curvature $-\frac{c}{4},[1]$.

\section{Curvature equations between a Hessian manifold and Its Submanifolds}

Let $M^{n}$ be an n-dimensional submanifolds of $(n+p)$ - dimensional Hessian manifold $M^{n+p}$. We choose a local field of orthonormal vectors such that $e_{1}, \ldots, e_{n}$ are tangent to $M^{n}$. We use the following convention on the ranges of indices: $1 \leq i, j, k, . . \leq n, n+1 \leq \alpha, \beta, \gamma, \ldots \leq p$.

We denote by $h_{i j}^{\alpha}$ the components of the second fundamental form of $M^{n}$ with respect to the frame $e_{1}, \ldots, e_{n}, \ldots, e_{n+p}$ the the mean curvature vector $\vec{H}$ of $M^{n}$, the square $H^{2}$ of the mean curvature $H$ of $M^{n}$ and the square $S$ of the length of the second fundamental form are given, respectively by

$$
\begin{gathered}
\vec{H}=\frac{1}{n} \sum_{\alpha=1}^{n} h^{\alpha} e_{\alpha} \text { where } h^{\alpha}=\sum_{i} h_{i i}^{\alpha} \\
H^{2}=\frac{1}{n^{2}} \sum_{\alpha=1}^{n}\left(h^{\alpha}\right)^{2} \\
S=\sum_{\alpha}\left[\sum_{i, j}^{n}\left(h_{i j}^{\alpha}\right)^{2}\right]=\sum_{\alpha} S_{\alpha} S_{\alpha},=\sum_{i, j}^{n}\left(h_{i j}^{\alpha}\right)^{2}
\end{gathered}
$$


$S$ and $H^{2}$ are independent of the choice of the orthonormal basis.

And for $S$ and $H^{2}$ we have

$$
\left(h_{\alpha}\right)^{2} \leq n S_{\alpha}, n H^{2} \leq S
$$

Let $X$ be an arbitrary unit vector tangent to $M^{n}$ at a point $x \in M^{n}$. We denote the the Ricci curvature of $M^{n}$ in the direction of $X$ by Ric $(X, X)$ and the Ricci curvature of $M^{n+p}$ in the some direction $X$ by $\overline{R i c}(X, X)$. Supposing that the local frame $e_{1}, \ldots, e_{n}, \ldots e_{n+p}$ is chosen that $e_{n}=X$. Then the Ricci curvature $\operatorname{Ric}(X, X)=\operatorname{Ric}\left(e_{n}, e_{n}\right)$ of $M^{n}$ at a point $x \in M^{n}$ is equal to

$$
\operatorname{Ric}\left(e_{n}, e_{n}\right)=\sum_{i=1}^{n-1} K_{\text {in }}
$$

where $K_{i n}$ is the sectional curvature of $M^{n}$ at $x \in M^{n}$ for the 2-plane section spanned by $e_{i}$ and $e_{n}$.

Let us consider $T_{x} M^{n}$ as an n-plane section of $M^{n+p}$ at a point $x \in M^{n}$ spanned by the $n$ - orthonormal vectors $e_{1}, \ldots, e_{n}$ then the $n$ - Ricci curvature $\overline{\operatorname{Ric}_{T_{x} M^{n}}}(X, X)$ of $M^{n+p}$ for the n-plane section $T_{x} M^{n}$ in the direction of $X=e_{n}$ is equal to

$$
\overline{\operatorname{Ric}_{T_{x} M^{n}}}(X, X)=\sum_{i=1}^{n-1} \overline{K_{i n}}
$$

where $\overline{K_{i n}}$ is the sectional curvature of $M^{n+p}$ at $x \in M^{n}$ for the 2-plane section spanned by the vectors $e_{i}$ and $e_{n}$.

Using Gauss equation

$$
R_{i j k l}=\bar{K}_{i j k l}+h_{i k}^{\alpha} h_{j l}^{\alpha}-h_{i l}^{\alpha} h_{j k}^{\alpha}
$$

then the $\operatorname{Ric}\left(e_{n}, e_{n}\right)$ of $M^{n}$ at $x \in M^{n}$ is equal to the following

$$
\operatorname{Ric}\left(e_{n}, e_{n}\right)=\sum_{i=1}^{n} \overline{K_{i n}}+\sum_{\alpha}\left[h_{n n}^{\alpha} \sum_{i=1}^{n} h_{i i}^{\alpha}-\sum_{i=1}^{n}\left(h_{i n}^{\alpha}\right)^{2}\right]
$$

or

$$
\operatorname{Ric}\left(e_{n}, e_{n}\right)=\overline{\operatorname{Ric}_{T_{X} M^{n}}}\left(e_{n}, e_{n}\right)+\sum_{\alpha}\left[h_{n n}^{\alpha} \sum_{i=1}^{n} h_{i i}^{\alpha}-\sum_{i=1}^{n}\left(h_{i n}^{\alpha}\right)^{2}\right]
$$

In [6] the author estimates for the Ricci curvature of a submanifold $M^{n}$ of an arbitrary Riemannian manifold $M^{n+p}$. After routine calculations we get similar results for Hessian manifolds as follows:

Theorem 3.1. Let $M^{n}$ be an $n$-dimensional submanifold of an $(n+p)$ dimensional Hessian manifold $M^{n+p}$. For the Ricci curvature Ric $(X, X)$ of $M^{n}$ at a non-totally geodesic point $x \in M^{n}$ in the direction of a unit vector $X$ tangent to $M^{n}$ we have

$$
\begin{aligned}
& \operatorname{Ric}(X, X) \leq \overline{\operatorname{Ric}_{T_{x} M^{n}}}(X, X)+\frac{n^{2} H^{2}}{4} \\
& \operatorname{Ric}(X, X) \geq \overline{\operatorname{Ric}_{T_{x} M^{n}}}(X, X)+(n-1) \sum_{\alpha} \lambda_{1}^{\alpha} \lambda_{n}^{\alpha} \\
&= \overline{\operatorname{Ric}_{T_{x} M^{n}}}(X, X)+ \\
& \frac{(n-1)}{n}\left[2 n H^{2}-S-(n-2) \sqrt{\frac{n H^{2}\left(S-n H^{2}\right)}{n-1}}\right]
\end{aligned}
$$

where $\lambda_{1}^{\alpha}$ and $\lambda_{n}^{\alpha}$ are two different eigenvalues of each one of the matrices $\left(h_{i j}^{\alpha}\right)$ of the second fundamental form. The equality in (3.10) is held only when all the matrices $\left(h_{i j}^{\alpha}\right)$ of the second fundamental form with respect to an orthonormal basis $e_{1}, \ldots, e_{n}=X, \ldots, e_{n+p}$ are of the form

$$
\left(\begin{array}{ccccc}
\widetilde{h}_{11}^{\alpha} & \widetilde{h}_{12}^{\alpha} & \ldots & \widetilde{h}_{1 n-1}^{\alpha} & 0 \\
\widetilde{h}_{12}^{\alpha} & \widetilde{h}_{22}^{\alpha} & \ldots & \widetilde{h}_{2, n-1}^{\alpha} & 0 \\
\vdots & \vdots & \vdots & \vdots & \vdots \\
\widetilde{h}_{1, n-1}^{\alpha} & \widetilde{h}_{2, n-1}^{\alpha} & \ldots & \widetilde{h}_{n-1, n-1}^{\alpha} & 0 \\
0 & 0 & \ldots & 0 & \frac{\widetilde{h}^{\alpha}}{2}
\end{array}\right)
$$


where $\widetilde{h}_{11}^{\alpha}+\widetilde{h}_{22}^{\alpha}+\ldots+\widetilde{h}_{n-1, n-1}^{\alpha}=\frac{\widetilde{h}_{\alpha}}{2}, \widetilde{h}_{i j}^{\alpha}-$ arbitrary $1 \leq i \leq j \leq n-1$.

The equality in (2.11) is fulfilled if and only if the matrices $\left(\widetilde{h}_{i j}^{\alpha}\right)$ are the following

$$
\left(\begin{array}{cccc}
\lambda_{1}^{\alpha} & 0 & \ldots & 0 \\
0 & \lambda_{1}^{\alpha} & \ldots & 0 \\
\vdots & \vdots & \ddots & \\
\ldots & \ldots & \ldots & \lambda_{n}^{\alpha}
\end{array}\right)
$$

where

$$
\lambda_{1}^{\alpha}=\frac{1}{n}\left(h^{\alpha} \mp \sqrt{\frac{n S_{\alpha}-\left(h_{\alpha}\right)^{2}}{n-1}}\right), \ldots, \lambda_{n}^{\alpha}=\frac{1}{n}\left(h^{\alpha} \mp(n-1) \sqrt{\frac{n S_{\alpha}-\left(h_{\alpha}\right)^{2}}{n-1}}\right)
$$

as $(n-1) \lambda_{1}^{\alpha}+\lambda_{n}^{\alpha}=h_{\alpha}$ and $(n-1)\left(\lambda_{1}^{\alpha}\right)^{2}+\left(\lambda_{n}^{\alpha}\right)^{2}=S_{\alpha}$.

For the proof of the Riemannian version of the theorem we refer to [6].

Corollary 3.1. If

$$
\frac{n^{2} H^{2}}{4} \leq-\overline{\operatorname{Ric}_{T_{x} M^{n}}}(X, X)
$$

then $\operatorname{Ric}(X, X) \leq 0$ at $x \in M^{n}$ for the unit vector $x \in T_{x} M^{n}$. Particularly when the ambient space $M^{n+p}$ is a space form on positive curvature then

$$
\operatorname{Ric}(X, X) \leq 0 \text { if } H^{2} \leq \frac{(n-1) c}{n^{2}} .
$$

Corollary 3.2. If

$$
2(n-1) H^{2}-\frac{n-1}{n} S-\frac{(n-2)}{n} \sqrt{n(n-1) H^{2}\left(S-n H^{2}\right)} \geq 0
$$

then $\operatorname{Ric}(X, X) \geq \overline{\operatorname{Ric}_{T_{x} M^{n}}}(X, X)$ at $x \in M^{n}$ in the direction of $x \in T_{x} M^{n}$.

Corollary 3.3. If

$$
\overline{\operatorname{Ric}_{T_{x} M^{n}}}(X, X) \geq 2(1-n) H^{2}+\frac{n-1}{n} S+\frac{n-2}{n} \sqrt{n(n-1) H^{2}\left(S-n H^{2}\right)} \geq 0
$$

then $\operatorname{Ric}(X, X) \geq 0$ for $x \in T_{x} M^{n}$. Next we may expess the following theorem

Theorem 3.2. Let $M^{n}$ be an n-dimensional submanifold isometrically immersed in an $(n+p)$ - dimensional arbitrary Hessian manifold $M^{n+p}$. The equality

$$
\operatorname{Ric}(X, X)=\overline{\operatorname{Ric}_{T_{x} M^{n}}}(X, X)+\frac{n-1}{n}\left[2 n H^{2}-S-(n-2) \sqrt{\frac{n H^{2}\left(S-n H^{2}\right)}{n-1}}\right]
$$

is fulfilled at a non-totally geodesic point $x \in M^{n}$ in the direction of a tangent unit vector $X \in T_{x} M^{n}$ if and only if the following conditions are satisfied:

$$
\text { i) } R_{\beta k l}^{\alpha}=\bar{K}_{\beta k l}^{\alpha} \text { at } x \in M^{n} \text {. }
$$

ii) Each one of the matrices $\left(h_{i j}^{\alpha}\right)$ of the second fundamental form of $M^{n}$ has exactly $(n-1)$ eigenvalues equal to $\lambda_{1}^{\alpha}$ and are equal to the corresponding $\lambda_{n}^{\alpha}$ from (3.13).

iii) The vector $X$ is their common eigenvector corresponding to their simple eigenvalue $\lambda_{n}^{\alpha}$. 


\section{Submanifold of a Hessian space form}

In the case when the ambient space $M^{n+p}$ is a space form with curvature $-\frac{c}{4}$, then we may compute the equation (3.10) and (3.11) as follows by using (3.17) and $i$ )

$$
\begin{gathered}
\operatorname{Ric}(X, X) \leq(1-n) \frac{c}{4}+\frac{n^{2} H^{2}}{4} \\
\operatorname{Ric}(X, X) \geq(1-n) \frac{c}{4}+\frac{n-1}{n}\left[2 n H^{2}-S-(n-2) \sqrt{\frac{n H^{2}\left(S-n H^{2}\right)}{n-1}}\right] \\
\operatorname{Ric}(X, X)=(n-1)\left[2 H^{2}-\frac{1}{n} S-\frac{(n-2)}{n} \sqrt{\frac{n H^{2}\left(S-n H^{2}\right)}{n-1}}-\frac{c}{4}\right]
\end{gathered}
$$

Applying Theorem 2.1 to an arbitrary submanifold $M^{n}$ of a space form $M^{n+p}\left(-\frac{c}{4}\right)$ gives the following

Theorem 4.1. Let $M^{n}$ be a non-totally geodesic submanifold isometrically immersed in a Hessian space form $M^{n+p}\left(-\frac{c}{4}\right)$. The equality

$$
\min _{X} \operatorname{Ric}(X, X)=(n-1)\left[2 H^{2}-\frac{1}{n} S-\frac{(n-2)}{n} \sqrt{\frac{n H^{2}\left(S-n H^{2}\right)}{n-1}}-\frac{c}{4}\right]
$$

when $X$ runs over all unit tangent vectors of $M^{n}$ at a point $x \in M^{n}$, holds for all points $x \in M^{n}$ if and only if the normal bundle of $M^{n}$ is flat, each one of the matrices $\left(h_{i j}^{\alpha}\right)$ has exactly $(n-1)$ eigenvalues equal to the corresponding $\lambda_{1}^{\alpha}$ and one equal to $\lambda_{n}^{\alpha}$ from (3.13) and the vector $X_{0}$, for which the minimum of Ric $(X, X)$ is achieved, is their common eigenvector corresponding to their simple eigenvalue $\lambda_{n}^{\alpha}$.

Now let us consider a Hessian domain $(\Omega, D, g=D d \varphi)$ in $\mathbb{R}^{n}$ of constant Hessian sectional curvature $c$ as indicated [1].

Proposition 4.1. The following Hessian domains are examples of spaces of constant Hessian sectional curvature 0.

(1) Euclidean space $\left(\mathbb{R}^{n}, D, g=D d\left(1 / 2 \sum_{i=1}^{n}\left(x^{i}\right)^{2}\right)\right)$.

(2) $\left(\mathbb{R}^{n}, D, g=D d\left(\sum_{i=1}^{n} e^{x^{i}}\right)\right)$.

Proposition 4.2. Let $c$ be positive real number and let $\Omega$ be given by

$$
\Omega=\left\{\left(x^{1}, \ldots, x^{n}\right) \in \mathbb{R}^{n} \mid x^{n}>\frac{c}{2} \sum_{i=1}^{n-1}\left(x^{i}\right)^{2}\right\},
$$

and let $\varphi$ be a smooth function on $\Omega$ defined by

$$
\varphi=-\frac{1}{c} \log \left\{x^{n}-\frac{1}{2} \sum_{i=1}^{n-1}\left(x^{i}\right)^{2}\right\} .
$$

Then $\left(\Omega, D, g=D^{2} \varphi\right)$ is a simply connected Hessian manifold of positive constant Hessian sectional curvature $c$.

Hence the following theorem can be proved as a consequence of the properties above.

It is really surprising that $(\Omega, g)$ is isometric to hyperbolic space form $\left(H\left(-\frac{c}{4}\right), g\right)$ of constant sectional curvature $-c / 4$;

$$
\begin{aligned}
H & =\left\{\left(\xi^{1}, \ldots, \xi^{n-1}, \xi^{n}\right) \in \mathbb{R}^{n} \mid \xi^{n}>0\right\} \\
g & =\frac{1}{\left(\xi^{n}\right)^{2}}\left\{\sum_{i=1}^{n}\left(d \xi^{i}\right)^{2}+\frac{4}{c}\left(d \xi^{n}\right)^{2}\right\} .
\end{aligned}
$$


Proposition 4.3. Let $\varphi$ be a smooth function on $\mathbb{R}^{n}$ defined by

$$
\varphi=-\frac{1}{c} \log \left(\sum_{A=1}^{n} e^{-c x^{A}}+1\right)
$$

where $c$ is a negative constant. Then $\left(\mathbb{R}^{n}, \widetilde{D}, g=\widetilde{D}^{2} \varphi\right)$ is a simply connected Hessian manifold of negative constant Hessian sectional curvature $c$. The Riemannian manifold $\left(\mathbb{R}^{n}, g\right)$ is isometric a domain of the sphere $\sum_{i=1}^{n+1} \xi_{A}^{2}=-\frac{4}{c}$ defined by $\xi_{A}>0$ for all $A,[1]$.

Then we re-formulate the (4.1)-(4.4) as follows:

Corollary 4.1. Let the ambient space be one of the following space indicated below

(1) Euclidean space $\left(\mathbb{R}^{n}, D, g=D d\left(1 / 2 \sum_{i=1}^{n}\left(x^{i}\right)^{2}\right)\right)$.

(2) $\left(\mathbb{R}^{n}, D, g=D d\left(\sum_{i=1}^{n} e^{x^{i}}\right)\right)$

then we get

$$
\operatorname{Ric}(X, X) \leq \frac{n^{2} H^{2}}{4}
$$

Corollary 4.2. If $(\Omega, g)$ is isometric to hyperbolic space form $\left(H\left(-\frac{c}{4}\right), g\right)$ of constant sectional curvature $-c / 4$;

$$
\begin{aligned}
H & =\left\{\left(\xi^{1}, \ldots, \xi^{n-1}, \xi^{n+p}\right) \in \mathbb{R}^{n+p} \mid \xi^{n+p}>0\right\}, \\
g & =\frac{1}{\left(\xi^{n+p}\right)^{2}}\left\{\sum_{i=1}^{n+p}\left(d \xi^{i}\right)^{2}+\frac{4}{c}\left(d \xi^{n}\right)^{2}\right\} .
\end{aligned}
$$

.Let us take ambient space as $M^{n+p}=(\Omega, g)$ then

$$
\operatorname{Ric}(X, X) \geq(1-n) \frac{c}{4}+\frac{n-1}{n}\left[2 n H^{2}-S-(n-2) \sqrt{\frac{n H^{2}\left(S-n H^{2}\right)}{n-1}}\right]
$$

and

$$
\operatorname{Ric}(X, X)=(n-1)\left[2 H^{2}-\frac{1}{n} S-\frac{(n-2)}{n} \sqrt{\frac{n H^{2}\left(S-n H^{2}\right)}{n-1}}-\frac{c}{4}\right] .
$$

Corollary 4.3. Let $\varphi$ be a smooth function on $\mathbb{R}^{n+p}$ defined by

$$
\varphi=-\frac{1}{c} \log \left(\sum_{A=1}^{n+p} e^{-c x^{A}}+1\right)
$$

where $c$ is a negative constant. Then $\left(\mathbb{R}^{n+p}, \widetilde{D}, g=\widetilde{D}^{2} \varphi\right)$ is isometric a domain of the sphere $\sum_{i=1}^{n+1} \xi_{A}^{2}=-\frac{4}{c}$ defined by $\xi_{A}>0$ for all $A$. If the ambient space is $\left(\mathbb{R}^{n+p}, \widetilde{D}, g=\widetilde{D}^{2} \varphi\right)$ we get

$$
\begin{gathered}
\operatorname{Ric}(X, X) \geq(n-1) \frac{c}{4}+\frac{n-1}{n}\left[2 n H^{2}-S-(n-2) \sqrt{\frac{n H^{2}\left(S-n H^{2}\right)}{n-1}}\right] \\
\operatorname{Ric}(X, X)=(n-1)\left[2 H^{2}-\frac{1}{n} S-\frac{(n-2)}{n} \sqrt{\frac{n H^{2}\left(S-n H^{2}\right)}{n-1}}+\frac{c}{4}\right] .
\end{gathered}
$$




\section{References}

[1] Shima, H. The Geometry of Hessian structures, World Scientific Publ., 2007.

[2] Shima, H., Homogeneous Hessian manifolds. Ann. Inst. Fourier, Grenoble,. 30, 3, (1980), 91-128.

[3] Shima, H., A differential geometric characterization of homogeneous sel-dual cones. Tsukuba J. Math Vol. 6, no.1, (1982), 79-88.

[4] Bektas, M., Yildirim, M., Integral inequalities for submanifolds of Hessian manifolds with constant Hessian sectional curvature. Iranian Journal of Sci. and Tech.Trans. A. Sci. vol.30, no.A2 (2006), 235-239.

[5] Yildirim Yilmaz,M., Bektas, M., A Survey on curvatures of Hessian manifolds. Chaos, Solitons and Fractals 38, (2008), 620-630.

[6] Hineva, S., Submanifolds for which a lower bound of the Ricci curvature is achieved. J.Geom. 88, (2008), 53-69.

[7] Cao,X.-F., Pseudo-umbilical submanifolds of constant curvature Riemannian manifolds, Glasgow Mathematical Journal vol.43,no. 1 (2001), 129-133.

[8] Yildirim Yilmaz, M. , Bektas, M. A Note on Pseudo-Umbilical Submanifolds of Hessian Manifolds with Constant Hessian Sectional Curvature International Scholarly Research Network ISRN Geometry Vol.2011, (2011), 1-12.

\section{Affiliations}

MÜNEVVER YILDIRIM YILMAZ

AdDRESS: Firat University, Dept. of Mathematics, 23119, Elazig-Turkey. E-MAIL: myildirim@firat.edu.tr

MEHMET BEKTAŞ

ADDRESS: Firat University, Dept. of Mathematics, 23119, Elazig-Turkey.

E-MAIL: mbektas@firat.edu.tr 